\title{
The Influence of age and sex on some blood parameters in healthy donkey in south Valley Egypt
}

\author{
A. E. Ahmed ${ }^{1}$, H. Y. Abdel-Hamid ${ }^{1}$, A. A. Abdel-Rahim ${ }^{2}$, M. N.Ismail ${ }^{1}$ \\ ${ }^{1}$ Department of Animal Medicine, Faculty of Veterinary Medicine, Qena, South Valley University \\ and ${ }^{2}$ Department Of Animal Medicine, Faculty of Veterinary Medicine, Assiut University, Egypt.
}

\begin{abstract}
A total number of 140 Egyptian breed donkeys were used in this study. Animals are classified into two groups according to the sex. Each group was further subdivided into four subgroups according to their ages. All animals were proved to be health by clinical and laboratory examinations. Two blood samples were collected from each donkey, one with anticoagulant and the other without anticoagulant for obtaining clear non-haemolysed serum. Various tests were conducted to measure the values of some blood contents. It was clear that total RBCs count, hemoglobin content and packed cells volume showed marked decrease with the increase of age. Significant difference in RBCs count between some groups and highly significant difference in $\mathrm{Hb}$ and PCV contents between another groups. Gradual elevation in the values of total leucocytes count from one month up to 10 years old was observed. Marked decrease in total WBCs count was reported in animals of both sexes on 10-20 years old. This denotes that Significant and highly significant differences appear in total WBCs count between animal groups. The biochemical parameters revealed highly significant difference in the total protein and albumin in some groups of male animals. Non significant fluctuation was observed in blood serum calcium, phosphorus and magnesium regarding the age and sex factors. In conclusion, it was clear that both age and sex factors has a marked influence on some blood contents in Egyptian donkeys.
\end{abstract}

The information regarding the contribution of draught animal power to the economics of developing countries is scare. Donkeys are better survived as draught due to their small size, ability to consume poor quality foods, lower feed and water requirements (Dijkman, 1995). It has been stated that the milk of lactating female donkeys is better than cows milk for human consumption (Salimei et al., 2004). Donkeys are one of the most under appreciated important draught animals in the worlds, serving a key role in the agricultural economy of the developing countries. In Egypt, donkeys are considered important animals for the farmers. They are used for carrying people and transporting goods. Zinkel et al., (1990); Paglia (2001) stated that sex and age factors in donkeys has significant on most of the blood picture in donkeys. French and Patrick (1995) observed that there were no significant differences for age or sex factors for any analyte in donkeys. Sato et al., (1979) Mentioned that most of blood biochemicals has a correlation to age and some of them to sex in donkeys.

The aim of the present work was to find out the possible correlation between some hematological and blood serum biochemical parameters and age and sex in native breed donkeys.

\section{Materials and methods}

Animals. Animals used in the present work were 140 donkeys of both sexes. Their ages ranged between one month and 20 years old. The animals were healthy and free from internal and external parasites as proved by clinical and Laboratory examinations. According to the sex and age, animals were divided into two groups.

Samples. Two blood samples were obtained from each animals one with EDTA as whole blood and the other for obtaining clear serum. Total Erythrocytes cells count, Hemoglobin content, packed cell and total white cells count were determined according to (Coles, 1986).

The blood serum samples were used for the estimation of total protein, albumin (Doumas, 1971), globulins by mathematical method and Also blood serum calcium, inorganic phosphorus and magnesium were determined according to the methods described by Gindler and King (1972); Morinal and Prox (1973); Gindler and Heth (1971) respectively - All the tests were done by using test kits and the values were obtained by spectrophotometer. The obtained data were subjected to statistical analysis (SPSSWIN, 1995).

\section{Results and Discussion}

The obtained results were summerized in tables $1,2,3,4,5$ and 6 . 
Table1: Effect of age on blood picture between male groups.

\begin{tabular}{|c|c|c|c|c|c|c|c|c|c|c|c|c|c|c|}
\hline \multirow{3}{*}{ parameters } & \multirow{2}{*}{\multicolumn{2}{|c|}{$\begin{array}{c}\text { Male Group } 1 \\
\text { NO. }=20\end{array}$}} & \multirow{2}{*}{\multicolumn{2}{|c|}{$\begin{array}{c}\text { Male Group } 2 \\
\text { NO. }=20\end{array}$}} & \multirow{2}{*}{\multicolumn{2}{|c|}{$\begin{array}{c}\text { Male Group } 3 \\
\text { NO. }=20\end{array}$}} & \multirow{2}{*}{\multicolumn{2}{|c|}{$\begin{array}{c}\text { Male Group } 4 \\
\text { NO. }=10\end{array}$}} & \multirow{2}{*}{\multicolumn{6}{|c|}{ Significant }} \\
\hline & & & & & & & & & & & & & & \\
\hline & Mean & SD. & Mean & SD. & Mean & S.D. & Mean & S.D. & $1 \mathrm{w} 2$ & $1 \mathrm{w} 3$ & $1 \mathrm{w} 4$ & $2 w 3$ & $2 w 4$ & $3 \mathrm{w} 4$ \\
\hline RBC, (T/L) & 8.34 & 0.42 & 8.31 & 0.42 & 8.24 & 0.76 & 8.21 & 0.21 & N.S. & N.S. & N.S. & N.S. & N.S. & N.S. \\
\hline $\mathrm{Hb},(\mathrm{g} / \mathrm{dl})$ & 12.40 & 0.74 & 12.25 & 0.96 & 12.10 & 1.00 & 12.00 & 0.71 & N.S. & N.S. & $*$ & N.S. & $*$ & $*$ \\
\hline PCV,$(\%)$ & 36.85 & 2.07 & 36.40 & 0.94 & 36.00 & 1.81 & 35.80 & 0.98 & N.S. & N.S. & $*$ & N.S. & $*$ & N.S. \\
\hline MCV, (fl) & 44.18 & 3.04 & 43.81 & 7.96 & 43.72 & 4.33 & 43.61 & 1.62 & N.S. & N.S. & N.S. & N.S. & $*$ & $*$ \\
\hline МСH, (pg) & 14.87 & 1.13 & 14.74 & 2.79 & 14.69 & 1.85 & 14.62 & 1.04 & N.S. & N.S. & $*$ & N.S. & $*$ & $*$ \\
\hline MCHC, (g/dl) & 33.65 & 1.48 & 33.65 & 3.36 & 33.61 & 3.28 & 33.52 & 1.47 & N.S. & N.S. & N.S. & N.S. & N.S. & N.S. \\
\hline \multicolumn{15}{|l|}{ ESR, (mm) } \\
\hline R110 (min.) & 3.50 & 1.29 & 3.8 & 1.1 & 4.45 & 0.947 & 5.2 & 0.4 & N.S. & $*$ & $* *$ & N.S. & $* *$ & $*$ \\
\hline R2 20 (min.) & 20.35 & 4.417 & 20.9 & 1.78 & 23.08 & 3.94 & 27.6 & 3.87 & N.S. & N.S. & $*$ & $* *$ & $* *$ & N.S. \\
\hline R3 30 (min.) & 36.42 & 4.79 & 38.5 & 4.11 & 42.7 & 4.44 & 45.0 & 4.47 & N.S. & $* *$ & $*$ & $* *$ & $*$ & N.S. \\
\hline R4 40 (min.) & 52.29 & 7.12 & 55.1 & 4.62 & 58.91 & 6.27 & 61.0 & 7.34 & N.S. & * & N.S. & $*$ & N.S. & N.S. \\
\hline Total WBC,(G/L) & 13.2 & 1.39 & 13.8 & 1.16 & 13.9 & 0.789 & 12.6 & 0.76 & N.S. & N.S. & N.S. & N.S. & $*$ & $*$ \\
\hline Seg. Neutrophil, (\%) & 61.57 & 4.01 & 61.8 & 2.45 & 62.23 & 1.97 & 63.6 & 2.059 & N.S. & N.S. & N.S. & N.S. & N.S. & N.S. \\
\hline Band cell, $(\%)$ & 1.14 & 0.914 & 1.33 & 6.99 & 0.972 & 0.944 & 1.2 & 0.748 & N.S. & N.S. & N.S. & N.S. & N.S. & N.S. \\
\hline Eosinophil,( \%) & 3.07 & 0.96 & 3.2 & 0.748 & 3.29 & 0.865 & 3.8 & 0.748 & N.S. & N.S. & N.S. & N.S. & N.S. & N.S. \\
\hline Basophil, (\%) & 0.785 & 0.557 & 1.33 & 0.788 & 1.37 & 0.748 & 1.4 & 0.489 & $*$ & $* *$ & N.S. & N.S. & N.S. & N.S. \\
\hline Lymphocyte, (\%) & 32.28 & 3.53 & 30.9 & 2.14 & 30.51 & 2.02 & 27.8 & 1.6 & N.S. & N.S. & $* *$ & N.S. & $*$ & $*$ \\
\hline Monocyte, (\%) & 1.14 & 0.742 & 1.33 & 0.699 & 1.54 & 0.64 & 2.2 & 0.4 & N.S. & N.S. & $* *$ & N.S. & $* *$ & $*$ \\
\hline
\end{tabular}


Table2: Effect of age on blood picture between female groups.

\begin{tabular}{|c|c|c|c|c|c|c|c|c|c|c|c|c|c|c|}
\hline \multirow{3}{*}{ parameters } & \multirow{2}{*}{\multicolumn{2}{|c|}{$\begin{array}{c}\text { female Group } 1 \\
\text { NO. }=\mathbf{2 0}\end{array}$}} & \multirow{2}{*}{\multicolumn{2}{|c|}{$\begin{array}{c}\text { Female Group } 2 \\
\text { NO. }=20\end{array}$}} & \multirow{2}{*}{\multicolumn{2}{|c|}{$\begin{array}{c}\text { Female Group } 3 \\
\text { NO. }=\mathbf{2 0}\end{array}$}} & \multirow{2}{*}{\multicolumn{2}{|c|}{$\begin{array}{c}\text { Female Group } 4 \\
\text { NO. }=10\end{array}$}} & \multirow{2}{*}{\multicolumn{6}{|c|}{ Significant }} \\
\hline & & & & & & & & & & & & & & \\
\hline & Mean & SD. & Mean & SD. & Mean & S.D. & Mean & S.D. & $1 \mathrm{w} 2$ & $1 \mathrm{w} 3$ & $1 \mathrm{w} 4$ & $2 \mathrm{w3}$ & $2 \mathrm{w} 4$ & 3 w 4 \\
\hline RBC, (T/L) & 7.80 & 0.62 & 7.79 & 0.39 & 7.65 & 0.30 & 7.50 & 0.28 & N.S. & N.S. & N.S. & N.S. & N.S. & N.S. \\
\hline $\mathrm{Hb},(\mathrm{g} / \mathrm{dl})$ & 11.00 & 0.41 & 10.80 & 0.43 & 10.50 & 0.40 & 10.00 & 0.33 & N.S. & N.S. & N.S. & N.S. & N.S. & * \\
\hline PCV, (\%) & 34.20 & 1.41 & 34.00 & 1.36 & 33.14 & 0.99 & 32.00 & 0.43 & N.S. & N.S. & N.S. & N.S. & N.S. & $*$ \\
\hline MCV, (fl) & 43.85 & 5.46 & 43.63 & 4.03 & 43.34 & 1.65 & 42.67 & 1.925 & N.S. & N.S. & N.S. & N.S. & N.S. & N.S. \\
\hline MCH, (pg) & 14.10 & 2.38 & 13.86 & 0.762 & 13.73 & 0.542 & 13.33 & 0.93 & N.S. & N.S. & N.S. & N.S. & N.S. & N.S. \\
\hline MCHC, (g/dl) & 32.16 & 2.34 & 31.76 & 2.02 & 31.68 & 1.18 & 31.25 & 0.92 & N.S. & N.S. & N.S. & N.S. & N.S. & N.S. \\
\hline \multicolumn{15}{|l|}{ ESR, (mm) } \\
\hline R1(10 min.) & 4 & 0.816 & 4.4 & 0.8 & 4.71 & 0.958 & 5.75 & 0.433 & N.S. & N.S. & N.S. & N.S. & $*$ & $*$ \\
\hline R2 (20 min.) & 21.66 & 2.35 & 22 & 2.44 & 23.92 & 3.36 & 28.3 & 2.046 & N.S. & N.S. & $*$ & N.S. & $* *$ & $*$ \\
\hline R3 (30 min.) & 40.66 & 0.942 & 42 & 6 & 45.71 & 5.28 & 46.3 & 6.495 & N.S. & $* *$ & N.S. & N.S. & N.S. & N.S. \\
\hline R4 (40 min.) & 53.33 & 4.714 & 60 & 6.32 & 62.5 & 5.26 & 63.8 & 2.165 & N.S. & N.S. & N.S. & N.S. & N.S. & N.S. \\
\hline Total WBC,(G/L) & 12.55 & 0.348 & 12.7 & 0.28 & 13.14 & 1.05 & 12 & 0.152 & N.S. & N.S. & N.S. & N.S. & $* *$ & $* *$ \\
\hline Seg. Neutrophil, (\%) & 59.33 & 4.71 & 60.2 & 2.71 & 61.07 & 2.404 & 62.3 & 0.433 & N.S. & N.S. & N.S. & N.S. & N.S. & N.S. \\
\hline Band cell, (\%) & 1.33 & 0.471 & 1.4 & 0.489 & 1.5 & 1.118 & 1.75 & 0.433 & N.S. & N.S. & N.S. & N.S. & N.S. & N.S. \\
\hline Eosinophil,( \%) & 3 & 0.816 & 3.8 & 0.748 & 4.07 & 0.457 & 4.25 & 0.829 & N.S. & N.S. & N.S. & N.S. & N.S. & N.S. \\
\hline Basophil, (\%) & 0.667 & 0.471 & 0.8 & 0.4 & 1.285 & 0.88 & 1.25 & 0.829 & N.S. & N.S. & N.S. & N.S. & N.S. & N.S. \\
\hline Lymphocyte, (\%) & 35 & 3.74 & 33.2 & 2.31 & 31.07 & 1.533 & 28.8 & 1.639 & N.S. & N.S. & N.S. & N.S. & $*$ & N.S. \\
\hline Monocyte, (\%) & 0.666 & 0.471 & 0.6 & 0.489 & 1.07 & 0.799 & 1.75 & 0.433 & N.S. & N.S. & N.S. & N.S. & $* *$ & N.S. \\
\hline
\end{tabular}


Table 3: Effect of sex on heamtological paramter in donkey.

\begin{tabular}{|c|c|c|c|c|c|c|c|c|c|c|c|c|c|c|c|c|c|c|c|c|}
\hline \multirow{4}{*}{ parameter } & \multirow{2}{*}{\multicolumn{2}{|c|}{$\begin{array}{c}\text { Male } \\
\text { Group1 }\end{array}$}} & \multicolumn{8}{|c|}{ Male } & & & \multicolumn{8}{|c|}{ Male Group } \\
\hline & & & \multicolumn{3}{|c|}{ Female Group1 } & \multicolumn{2}{|c|}{ Group2 } & \multicolumn{3}{|c|}{ Female Group2 } & \multicolumn{2}{|c|}{ Male Group3 } & \multicolumn{3}{|c|}{ Female Group3 } & \multicolumn{2}{|c|}{4} & \multicolumn{3}{|c|}{ Female Group 4} \\
\hline & \multicolumn{2}{|c|}{ NO. $=\mathbf{2 0}$} & \multicolumn{3}{|c|}{ NO. $=20$} & \multicolumn{2}{|c|}{ NO. $=20$} & \multicolumn{3}{|c|}{ NO. $=20$} & \multicolumn{2}{|c|}{ NO. $=20$} & \multicolumn{3}{|c|}{ NO. $=20$} & \multicolumn{2}{|c|}{ NO. $=10$} & \multicolumn{3}{|c|}{ NO. $=10$} \\
\hline & Mean & SD. & Mean & SD. & $\mathbf{p}$ & Mean & S.D. & Mean & S.D. & $\mathbf{p}$ & Mean & S.D. & Mean & S.D. & $\mathbf{p}$ & Mean & S.D. & Mean & S.D. & $\mathbf{P}$ \\
\hline RBC, (T/L) & 8.34 & 0.42 & 7.80 & 0.62 & N.S. & 8.31 & 0.42 & 7.79 & 0.39 & N.S. & 8.24 & 0.76 & 7.65 & 0.30 & $* *$ & 8.21 & 0.21 & 7.50 & 0.28 & $* *$ \\
\hline $\mathrm{Hb},(\mathrm{g} / \mathrm{dl})$ & 12.40 & 0.74 & 11.00 & 0.41 & N.S. & 12.25 & 0.96 & 10.80 & 0.43 & $* *$ & 12.10 & 1.00 & 10.50 & 0.40 & $* *$ & 12.00 & 0.71 & 10.00 & 0.33 & $* *$ \\
\hline PCV,(\%) & 36.85 & 2.07 & 34.20 & 1.41 & N.S. & 36.40 & 0.94 & 34.00 & 1.36 & $* *$ & 36.00 & 1.81 & 33.14 & 0.99 & $* *$ & 35.80 & 0.98 & 32.00 & 0.43 & $* *$ \\
\hline MCV, (fl) & 44.18 & 3.04 & 43.85 & 5.46 & N.S. & 43.81 & 7.96 & 43.63 & 4.03 & N.S. & 43.72 & 4.33 & 43.34 & 1.65 & N.S. & 43.61 & 1.62 & 42.67 & 1.93 & N.S. \\
\hline МCH, (pg) & 14.87 & 1.13 & 14.10 & 2.38 & * & 14.74 & 2.79 & 13.86 & 0.76 & * & 14.69 & 1.85 & 13.73 & 0.54 & $* *$ & 14.62 & 1.04 & 13.33 & 0.93 & N.S. \\
\hline $\mathrm{MCHC},(\mathrm{g} / \mathrm{dl})$ & 33.65 & 1.48 & 32.16 & 2.34 & N.S. & 33.65 & 3.36 & 31.76 & 2.02 & N.S. & 33.61 & 3.28 & 31.68 & 1.18 & $* *$ & 33.52 & 1.47 & 31.25 & 0.92 & N.S. \\
\hline \multicolumn{21}{|l|}{ ESR, (mm) } \\
\hline R1 (10 min.) & 3.50 & 1.29 & 4.00 & 0.82 & N.S. & 3.80 & 1.10 & 4.40 & 0.80 & N.S. & 4.45 & 0.95 & 4.71 & 0.96 & N.S. & 5.20 & 0.40 & 5.75 & 0.43 & N.S. \\
\hline R2 (20 min.) & 20.35 & 4.42 & 21.66 & 2.35 & N.S. & 20.86 & 1.78 & 22.00 & 2.44 & N.S. & 23.08 & 3.94 & 23.92 & 3.36 & N.S. & 27.60 & 3.87 & 28.25 & 2.05 & N.S. \\
\hline R3 (30 min.) & 36.42 & 4.79 & 40.66 & 0.94 & $*$ & 38.53 & 4.11 & 42.00 & 6.00 & N.S. & 42.70 & 4.44 & 45.71 & 5.28 & N.S. & 45.00 & 4.47 & 46.25 & 6.50 & N.S. \\
\hline R4 (40 min.) & 52.29 & 7.12 & 53.33 & 4.71 & N.S. & 55.06 & 4.62 & 60.00 & 6.32 & N.S. & 58.91 & 6.27 & 62.50 & 5.26 & N.S. & 61.00 & 7.34 & 63.75 & 2.17 & N.S. \\
\hline WBC, $(\mathrm{G} / \mathrm{L})$ & 13.2 & 1.39 & 12.55 & 0.348 & N.S. & 13.76 & 1.16 & 12.72 & 0.28 & $* *$ & 13.9 & 0.7886 & 13.14 & 1.05 & * & 12.62 & 0.76 & 12.02 & 0.152 & N.S. \\
\hline $\begin{array}{c}\text { Seg. neutrophil, } \\
(\%)\end{array}$ & 61.57 & 4.01 & 59.33 & 4.71 & N.S. & 61.8 & 2.45 & 60.2 & 2.71 & N.S. & 62.2 & 1.97 & 61.07 & 2.404 & N.S. & 63.6 & 2.059 & 62.25 & 0.433 & N.S. \\
\hline Band cell (\%) & 1.14 & 0.914 & 1.33 & 0.471 & N.S. & 1.33 & 6.99 & 1.4 & 0.489 & N.S. & 0.97 & 0.944 & 1.5 & 1.118 & N.S. & 1.2 & 0.748 & 1.75 & 0.433 & N.S. \\
\hline \multicolumn{21}{|l|}{ Eosinophil, } \\
\hline$(\%)$ & 3.07 & 0.96 & 3 & 0.816 & N.S. & 3.2 & 0.748 & 3.8 & 0.748 & N.S. & 3.29 & 0.865 & 4.07 & 0.457 & $* *$ & 3.8 & 0.748 & 4.25 & 0.829 & N.S. \\
\hline Basophil, (\%) & 0.785 & 0.557 & 0.667 & 0.471 & N.S. & 1.33 & 0.788 & 0.8 & 0.4 & N.S. & 1.37 & 0.748 & 1.285 & 0.88 & N.S. & 1.4 & 0.489 & 1.25 & 0.829 & N.S. \\
\hline \multicolumn{21}{|l|}{ Lymphocyte, } \\
\hline$(\%)$ & 32.28 & 3.53 & 35 & 3.74 & N.S. & 30.93 & 2.14 & 33.2 & 2.31 & N.S. & 30.5 & 2.02 & 31.07 & 1.533 & N.S. & 27.8 & 1.6 & 28.75 & 1.639 & N.S. \\
\hline Monocyte, (\%) & 1.14 & 0.742 & 0.666 & 0.471 & N.S. & 1.33 & 0.699 & 0.6 & 0.489 & $*$ & 1.54 & 0.64 & 1.07 & 0.7985 & N.S. & 2.2 & 0.4 & 1.75 & 0.433 & N.S. \\
\hline
\end{tabular}


Table 4: Effect of age on some biochemical parameters between male groups.

\begin{tabular}{|c|c|c|c|c|c|c|c|c|c|c|c|c|c|c|}
\hline \multirow{4}{*}{ parameters } & \multirow{2}{*}{\multicolumn{2}{|c|}{$\begin{array}{c}\text { Male Group } \\
1\end{array}$}} & \multirow{2}{*}{\multicolumn{2}{|c|}{$\begin{array}{c}\text { Male Group } \\
2\end{array}$}} & \multirow{2}{*}{\multicolumn{2}{|c|}{$\begin{array}{c}\text { Male Group } \\
\mathbf{3}\end{array}$}} & \multirow{2}{*}{\multicolumn{2}{|c|}{$\begin{array}{c}\text { Male Group } \\
4\end{array}$}} & \multirow{2}{*}{\multicolumn{6}{|c|}{ Significant }} \\
\hline & & & & & & & & & & & & & & \\
\hline & \multicolumn{2}{|c|}{ NO. $=\mathbf{2 0}$} & \multicolumn{2}{|c|}{ NO. $=\mathbf{2 0}$} & \multicolumn{2}{|c|}{ NO. $=20$} & \multicolumn{2}{|c|}{ NO. $=10$} & \multirow[b]{2}{*}{$1 \mathrm{w} 2$} & \multirow[b]{2}{*}{$1 \mathrm{w} 3$} & \multirow[b]{2}{*}{$1 \mathrm{w} 4$} & \multirow[b]{2}{*}{$2 \mathrm{w3}$} & \multirow[b]{2}{*}{$2 w 4$} & \multirow[b]{2}{*}{$3 \mathbf{w 4}$} \\
\hline & Mean & SD. & Mean & S.D. & Mean & S.D. & Mean & S.D. & & & & & & \\
\hline Total Protein (g/dl) & 7.73 & 1.252 & 7.86 & 0.509 & 7.86 & 0.604 & 8.01 & 0.567 & N.S. & N.S. & N.S. & N.S. & * & N.S. \\
\hline Albumin (g/dl) & 3.14 & 0.241 & 3.33 & 0.483 & 3.39 & 0.393 & 3.58 & 0.483 & N.S. & $*$ & N.S. & N.S. & $* *$ & $*$ \\
\hline Globulin (g/dl) & 4.59 & 1.174 & 4.53 & 0.673 & 4.48 & 0.634 & 4.43 & 0.676 & N.S. & N.S. & N.S. & N.S. & N.S. & N.S. \\
\hline $\mathrm{A} / \mathrm{G}$ ratio & 0.75 & 0.301 & 0.76 & 0.204 & 0.78 & 0.164 & 0.84 & 0.225 & N.S. & N.S. & N.S. & N.S. & N.S. & N.S. \\
\hline Calcium (mg/dl) & 9.34 & 1.279 & 9.45 & 0.064 & 9.51 & 0.72 & 9.63 & 1.41 & N.S. & N.S. & N.S. & N.S. & N.S. & N.S. \\
\hline Phosphorus (mg/dl) & 5.35 & 1.253 & 5.05 & 1.507 & 5.01 & 0.519 & 4.85 & 0.519 & N.S. & N.S. & N.S. & N.S. & N.S. & N.S. \\
\hline Magnesium (mg/dl) & 2.3 & 0.308 & 2.49 & 0.314 & 2.61 & 0.404 & 2.81 & 0.557 & N.S. & $* *$ & N.S. & N.S. & N.S. & N.S. \\
\hline
\end{tabular}

Table 5: Effect of age on some biochemical parameters between female groups.

\begin{tabular}{|c|c|c|c|c|c|c|c|c|c|c|c|c|c|c|}
\hline \multirow{3}{*}{ parameters } & \multirow{2}{*}{\multicolumn{2}{|c|}{$\begin{array}{l}\text { Female } \\
\text { Group } 1 \\
\text { NO.=20 }\end{array}$}} & \multirow{2}{*}{\multicolumn{2}{|c|}{$\begin{array}{l}\text { Female } \\
\text { Group } 1 \\
\text { NO.=20 }\end{array}$}} & \multirow{2}{*}{\multicolumn{2}{|c|}{$\begin{array}{l}\text { Female } \\
\text { Group } 2 \\
\text { NO. }=20\end{array}$}} & \multirow{2}{*}{\multicolumn{2}{|c|}{$\begin{array}{l}\text { Female } \\
\text { Group } 3 \\
\text { NO.=10 }\end{array}$}} & \multirow{2}{*}{\multicolumn{6}{|c|}{ Significant }} \\
\hline & & & & & & & & & & & & & & \\
\hline & Mean & SD. & Mean & SD. & Mean & S.D. & Mean & S.D. & $1 \mathrm{w} 2$ & $1 \mathrm{w} 3$ & $1 \mathrm{w} 4$ & $2 w 3$ & $2 w 4$ & $3 w 4$ \\
\hline Total Protein (g/dl) & 7.09 & 0.851 & 7.13 & 0.134 & 7.29 & 0.708 & 7.69 & 0.296 & N.S. & N.S. & N.S. & N.S. & N.S. & N.S. \\
\hline Albumin (g/dl) & 3.43 & 0.56 & 3.54 & 0.3 & 3.63 & 0.667 & 4.17 & 0.126 & N.S. & N.S. & N.S. & N.S. & N.S. & N.S. \\
\hline Globulin (g/dl) & 4.15 & 0.362 & 3.59 & 0.402 & 3.66 & 0.947 & 3.52 & 0.341 & N.S. & N.S. & N.S. & N.S. & N.S. & N.S. \\
\hline A/G ratio & 0.84 & 0.187 & 1.01 & 0.211 & 1.09 & 0.398 & 1.2 & 0.14 & N.S. & N.S. & N.S. & N.S. & N.S. & N.S. \\
\hline Calcium (mg/dl) & 8.46 & 1.046 & 8.74 & 2.077 & 9.02 & 1.61 & 9.26 & 2.027 & N.S. & N.S. & N.S. & N.S. & N.S. & N.S. \\
\hline Phosphorus (mg/dl) & 5.25 & 0.204 & 5.03 & 1.007 & 4.99 & 1.124 & 4.96 & 0.752 & N.S. & N.S. & N.S. & N.S. & N.S. & N.S. \\
\hline Magnesium (mg/dl) & 2.45 & 0.238 & 2.56 & 0.127 & 2.59 & 0.214 & 2.64 & 0.099 & N.S. & N.S. & N.S. & N.S. & N.S. & N.S. \\
\hline
\end{tabular}


Table 6: Effect of sex on biochemical parameters in donkey.

\begin{tabular}{|c|c|c|c|c|c|c|c|c|c|c|c|c|c|c|c|c|c|c|c|c|}
\hline \multirow{3}{*}{ parameter } & \multirow{2}{*}{\multicolumn{2}{|c|}{$\begin{array}{c}\text { Male Group } 1 \\
\text { NO. }=\mathbf{2 0}\end{array}$}} & \multicolumn{3}{|c|}{ Female Group 1} & \multicolumn{2}{|c|}{ Male Group 2} & \multicolumn{3}{|c|}{ Female Group 2} & \multicolumn{2}{|c|}{ Male Group 3} & \multicolumn{3}{|c|}{ Female Group 3} & \multicolumn{2}{|c|}{ Male Group 4} & \multicolumn{3}{|c|}{ Female Group 4} \\
\hline & & & & O. $=20$ & & NO. & & & $0 .=20$ & & NO & & & $O .=20$ & & NO & & & $0 .=10$ & \\
\hline & Mean & S.D. & Mean & S.D. & $\mathbf{P}$ & Mean & S.D. & Mean & S.D. & $\mathbf{P}$ & Mean & S.D. & Mean & S.D. & $\mathbf{P}$ & Mean & S.D. & Mean & S.D. & $\mathbf{P}$ \\
\hline $\begin{array}{l}\text { Total Protein } \\
\text { (g/dl) }\end{array}$ & 7.73 & 1.25 & 7.09 & 0.85 & N.S. & 7.86 & 0.51 & 7.13 & 0.13 & $* *$ & 7.86 & 0.60 & 7.29 & 0.708 & $*$ & 8.01 & 0.57 & 7.69 & 0.30 & N.S. \\
\hline $\begin{array}{c}\text { Albumin } \\
\text { (g/dl) }\end{array}$ & 3.14 & 0.24 & 3.43 & 0.56 & N.S. & 3.33 & 0.48 & 3.54 & 0.30 & N.S. & 3.39 & 0.39 & 3.63 & 0.667 & $* *$ & 3.58 & 0.48 & 4.17 & 0.13 & N.S. \\
\hline $\begin{array}{c}\text { Globulin } \\
\text { (g/dl) }\end{array}$ & 4.59 & 1.17 & 4.15 & 0.36 & N.S. & 4.53 & 0.67 & 3.59 & 0.40 & $* *$ & 4.48 & 0.63 & 3.66 & 0.947 & $* *$ & 4.43 & 0.68 & 3.52 & 0.34 & $*$ \\
\hline A/G ratio & 0.75 & 0.30 & 0.84 & 0.19 & N.S. & 0.76 & 0.20 & 1.01 & 0.21 & N.S. & 0.78 & 0.16 & 1.09 & 0.398 & $* *$ & 0.84 & 0.23 & 1.2 & 0.14 & N.S. \\
\hline $\begin{array}{c}\text { Calcium } \\
\text { (mg/dl) }\end{array}$ & 9.34 & 1.28 & 8.46 & 1.05 & N.S. & 9.45 & 0.06 & 8.74 & 2.08 & N.S. & 9.51 & 0.72 & 9.02 & 1.61 & N.S. & 9.63 & 1.41 & 9.26 & 2.03 & N.S. \\
\hline $\begin{array}{c}\text { Phosphorus } \\
\text { (mg/dl) }\end{array}$ & 5.35 & 1.25 & 5.25 & 0.20 & N.S. & 5.05 & 1.51 & 5.03 & 1.01 & N.S. & 5.01 & 0.52 & 4.99 & 1.124 & N.S. & 4.85 & 0.52 & 4.96 & 0.75 & N.S. \\
\hline $\begin{array}{l}\text { Magnesium } \\
\text { (mg/dl) }\end{array}$ & 2.3 & 0.31 & 2.45 & 0.24 & N.S. & 2.49 & 0.31 & 2.56 & 0.13 & N.S. & 2.61 & 0.40 & 2.59 & 0.2144 & N.S. & 2.81 & 0.56 & 2.64 & 0.10 & N.S. \\
\hline
\end{tabular}


Clinical laboratory diagnosis has an integral role in practice of veterinary Medicine and the availability of tests is important to clinician as are the history and clinical examination (Coles, 1986). Many of the investigated animals are owned by poor people and work in harsh environment, thus the study and recorded the healthy parameters of donkeys seemed to be important.

The obtained result (Tables 1,2) showed that the total red blood cells count were similar to those described by (Nayeri,1978) for both male and female Iranian donkeys, and higher than those reported by Enio et al., (2004) in Brazilian donkeys; AL - Busadah and Homeida (2005) in Hassawi Asses.

The reported hemoglobin values as shown tables were closely related to the values reported by Enio et al., (2004) in Barazilian donkeys; Al - Busadah and Hameida (2005) in Hassawi Asses, but higher than the result reported in donkeys by (Zinkel, et al., 2005).

The reported values of PCV in examined animals were the same as that reported by folch et al., (1997) in Catalonian donkeys, lower than Zinkel et al., (1990) in us donkeys and higher than French and Patrick (1995) in UK donkeys.

Our data on the total white blood cells count were in agreement with those previously reported by Nayeri (1978) in male Iranian donkeys but not for the Females. ON the other hand, our data on WBCs count were higher than those of Enio et al., (2004); Al-Busadah and Homeida (2005) in Brazilian and Hassawi Asses respectively.

The differences of analyses were due to differences in the feeding conditions among breads of donkeys, the climatic changes as well as the physiological states as pregnancy and Lactation factors (Kaneko, et al., 1997). Our study revealed that age has an effect on the hematological picture where all the studied parameters decreased with the increase of age. Similar results were reported in other breads of donkeys (Zinkell et al., 1990). On the other hand, Marco et al., (2005) reported that Red cells values were greater in donkeys under one year then the adult ones.

It was clear that the sex in donkeys has an effect on the hematological picture. Our data revealed that the values of all parameters were higher in male donkeys as compared with the female ones. Some parameters has Statistical difference, while others showed difference without statistical Significance our results were in contrast with that reported by French and Patrick (1995) .

Our data of some organic and inorganic blood serum constituents were seen in tables 4,5 where albumin in the blood of Egyptian donkeys were similar to that in U.S. donkeys ( Zinkl et al., 1990 ) and Indian donkeys (Gupta et al., 1994 ) . In the same time,

Enio et al., (2004) reported lower values in their donkeys. Enio et al., (2003) stated that physiological variations of serum protein and albumin can occur because of national influences hormonal effects, stress and guild loss by sweat

Slight non- Significant change was reported in the most studied biochemicals in blood serum of Egyptian donkeys (tables 4, 5 and 6)

Dinevv and khubenov (1986) found that the amount of total serum protein in young donkeys was lower than in older ones. It is also stated that the concentration of calcium and phosphorus was in reverse correlation with the age of animals. Phosphorus is higher and calcium is lower in young animals, but a very significant decrease with age observed for phosphors concentration in Catalonian donkeys (Folch et al., 1977; us donkeys Zinkell, et al., 1990).

Phosphorus decreased bare Metabolism as animals become older ( kaneko etal , 1997 ) Growth hormones were seemed largely responsible for the increase of phosphorus in young growing animals as it increase rural tubular resumption of phosphorus ( Stock ham and Michael , 2002 ).

In this work, sex has effect on some blood serum biochemicals at certain age. Similar findings were previously reported by Nayeri (1978); Cubeddu, et al., (1991) Who .recorded differences between female and male donkeys in serum protein and Albumin concentrations . On the other hand, French and Patrick (1995) reported non-significant differences for most of the blood biochemicals in relation to sex.

\section{Conclusion}

The total RBCs count, hemoglobin content and packed cells volume showed marked decrease with the increase of age. Significant difference in RBCs count between some groups and highly significant difference in $\mathrm{Hb}$ and $\mathrm{PCV}$ contents between other groups. Increase in total leucocytes count from one month up to 10 years old, was observed. Marked decrease in total WBCs count in animals of both sexes on 10-20 years old. Significant and highly significant differences appear in total WBCs count between 
animal groups. The biochemical parameters revealed highly significant difference in the total protein and albumin in some groups of male animals. Non significant fluctuation was observed in blood serum calcium, phosphorus and magnesium regarding the age and sex factors. It was clear that both age and sex factors has a marked influence on some blood contents in Egyptian donkeys.

\section{References}

AL-Busadah, K. A. and Homeida, A. M. (2005): Some Physical Variables, Biochemical and Haematological Parameters in Hassawi Ass. Sci., J. King Faisal Univ., (Basic and Applied Sciences) 6 (1):145-152.

Coles, E. H. (1986): Veterinary Clinical pathology; $4^{\text {th }}$ ed. W.B. Saunders Company. pp.8.

Cubeddu, G. M.; Bini, P. P.; Floris. B.; Carcangiu, V.; Bomboi, G. and Pintori, G. (1991): Hematologic parameters of the white donkeys of Asinara. Boll Soc. Ital. Biol. Sper., 67 (6): 577-84.

Dijkman, J. T. (1992): A note on the influence of negative pulling load .Animal Producing 54:153 -156.

Dinevv, D. and Khubenov. (1986): Normal values of hematolgical, biochemical and enzmatological indces the donkey. Vet. Med., 23 (10):69-75.

Doumas, B. (1971): Biochemical determination of albumin concentration. Clin. Chem. Acta, 31:87.

Enio M.; Fernandes, W. R.; Mirandola, R. M. S. ; Guilherme Kubo, Ferreira, R. R.; Oliveira, J. V. and Gacek, F. (2003): Reference Values on Serum Biochemical Parameters of Brazilian Donkey (Equus asinus) Breed. J. Equine Vet. Sci., 23 (8): 358 - 364

Enio M.; Mirandala, R. M. S. ;Ferreria, R.R. ;Oliveira, J. V. Francisco Gacek. And Fernandes, W. R.(2004): Reference values on hematologic parameters of the Brazilian donkey (Equus asinus) breed. J. Equine Vet. Sci., 24: 271- 276.

Folch, F.; Jonathan, J. and Cuenca, R. (1997): Reference rang and the influence of age and sex on haematological values of the endangered Catalion donkey. Vet. J., 154: 163-168.

French, J. M. and Patrick, V. H. (1995): Reference values for physiological, haematological and biochemical parameters in domestic donkey ( equus asinus). Equine Vet. Edu., 7: 33-50.

Ginder, E. and Heth, D. (1971): Determination of magnesium in serum and urine. Clin. Chem., 17:662.

Gindler, E. M. and King, J. D. (1972): Rapid colourimetric determination of calcium in biological fluid with thymol blue. Am. J. Clin. Path., 58: 376-382.

Gupta, A.K.; Varshiney, J. P.; Uppal, P. K. (1994): Comparative studies on biochemical indices in different breeds of equine. Idian Vet. J. 74:26-30.

Jonathan, S. O. and David, A. J. (1997): The erythrocyte sedimentation rate. J. Emerg. Med., 15, (6) : 869-874.

Kaneko, J. J.; Harvey, J. W. and Bruss, M. L. (1997): Clinical Biochemistry of Domestic Animals. Academic Press. pp. 124-128.

Marco C.; Tommaso, F.; Solano-Galleg D. L. ; Carli E. ; Tasca, S. and Lubas, G. (2005): Reference ranges for haematology, biochemical profile and electrophoresis in a single herd of Ragusana donkeys from Sicily (Italy) Comparative Clinical Pathology., Springer- London Limited 14(1) : 5-12.

Morinal, L. and Prox, J. (1973): New and rapid procedure for serum phosphorus using O-phenyl-Lendiamine as reductants. Clin. Chem. Ac. 469:112-117

Nayeri, G.D. (1978): Blood characteristics of the adult donkey. Zbl. Vet. Med. A., 25(55):1-7

Paglia, D. E. (2001): Radiomatrix assessment of hexomonphosphate shunk capacity in erythrocytes of Rhinocerus. Am. J. Vet. Res., 62: 1113-117.

Perdigo de Olivera; Augusto, C.; Grasso, P.L.; Souza, H. and Baudet, G. J. A. (1974): Eritrograma normal de jumentos Equus Asinus das ragas purosangue Italiana Brasileria de 1 a 2 anos de idade. B industry anim. 31: 3259

Pritchard, J. C.; Lindberg, A. C,; Main, D. C. J. and Whay, H. R. (2005): Assessment of the welfare of working horses, mules and donkeys, using health and behaviour parameters .Preventive veterinary medicine. 11 march 2005. Salimei, E.; Fantuz, F. ; Coppola, R. (2004):Composition and characteristics of asss milk. Anim. Res., 53: $67-78$.

Sato, O. K. (1979): Hematological and biochemical values of thorouh breed foals in the first six months of life. Covnell. Vet. Jan, 69 (1): $3-19$.

SPSSWIN (1995): Soft ware program for statistical analysis, USA.

Stockham, S.L. and Michael, A.S. (2002): Veterinary Clinical Pathology. $1^{\text {st }}$ Lowa state press ,Ablack well Publishing Company. pp.417-419.

Unkel, M. (1984): Concentration of k. Calcium, Magnesium, sodium and phosphate in blood serum from Iceland ponies. Tierarztiliche um Schau. 39:989 - 994.

Zinkl,G. J. ; Mae, D. ; Merida, P. G. ; Forva, T. B. and Humble, J. A. (1990) : Reference ranges and the influence of age and sex on hematologic and serum biochemical values in donkeys (Equus asinus Am. J. Vet. Res., 51(3): 408- 41 .

$$
\begin{aligned}
& \text { تم فى هذه الدراسة فحص عدد مائة و أربعين حماراً، وهذه الحمر قسمت إلى قسمين حسب الجنس، ثم قسمت الأكور }
\end{aligned}
$$

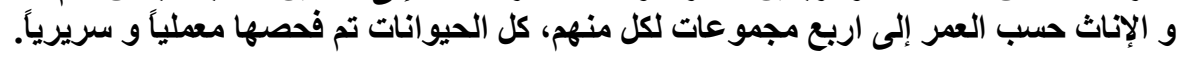

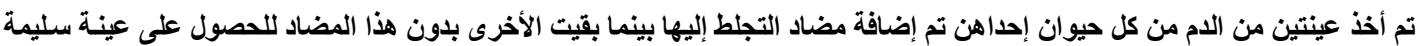

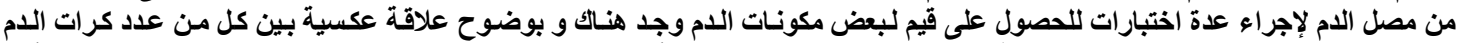

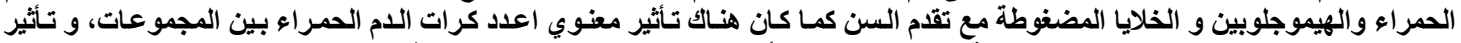

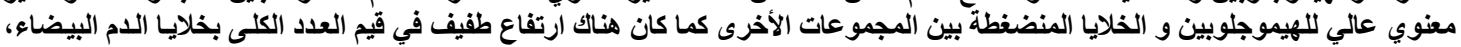

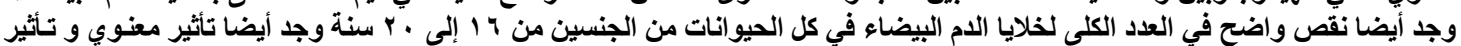

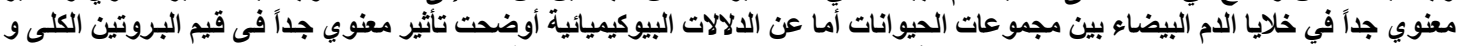

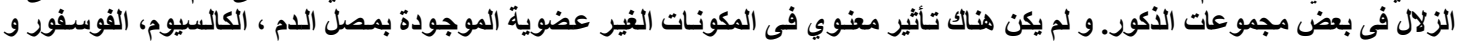

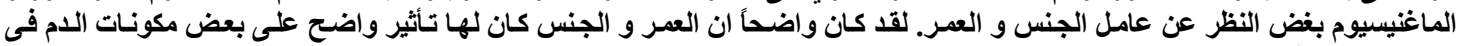

$$
\begin{aligned}
& \text { الحمير المصرية. }
\end{aligned}
$$


